\title{
Throwing and Catching as Relational Skills in Game Play: Situated Learning in a Modified Game Unit
}

\author{
Ann MacPhail,, ${ }^{1}$ David Kirk, ${ }^{2}$ and Linda Griffin ${ }^{3}$ \\ 'University of Limerick, ${ }^{2}$ Leeds Metropolitan University, \\ and ${ }^{3}$ University of Massachusetts
}

\begin{abstract}
In this article, we were interested in how young people learn to play games within a tactical games model (TGM) approach (Griffin, Oslin, \& Mitchell, 1997) in terms of the physical-perceptual and social-interactive dimensions of situativity. Kirk and MacPhail's (2002) development of the Bunker-Thorpe TGfU model was used to conceptualize the nature of situated learning in the context of learning to play an invasion game as part of a school physical education program. An entire class of 29 Year-5 students (ages 9-10 years) participated in a 12-lesson unit on an invasion game, involving two 40-min lessons per week for 6 weeks. Written narrative descriptions of videotaped game play formed the primary data source for the principal analysis of learning progression. We examined the physical-perceptual and social-interactive dimensions of situated learning (Kirk, Brooker, \& Braiuka, $2000)$ to explore the complex ways that students learn skills. Findings demonstrate that for players who are in the early stages of learning a ball game, two elementary, or fundamental, skills of invasion game play — throwing and catching a ball—are complex, relational, and interdependent.
\end{abstract}

Keywords: tactical games model, physical education, theory of learning

Recent developments in the fields of educational learning theory (e.g., Cobb \& Bowers, 1999) and research on expertise in sport (e.g., French \& McPherson, 1999) have provided researchers in physical education with new theoretical frameworks and tools with which to conceptualize learning. An emerging line of research has sought to use recent developments in learning theory as a means of reforming teaching and curriculum within physical education, working with theoretical frameworks from cognitive (Dodds, Griffin, \& Placek, 2001) and situated learning (Rovegno, Nevett, \& Babiarz, 2001a) perspectives.

Ann MacPhail is with the Department of Physical Education and Sport Sciences, University of Limerick, Limerick, Ireland; Kirk is with the Carnegie Faculty of Sport and Education, Leeds Metropolitan University, Leeds, U.K; and Griffin is with the School of Education, University of Massachusetts, Amherst, MA. 
Rink (1999) advocated that any reform of the curriculum should be based in a theory of learning. Curriculum development in physical education has rarely been informed by research on learning. Rink (1999) argued that the convergence of research on learning and curriculum has the potential to reform the pervasive use of the traditional or multiactivity model in physical education. Ennis (1999) has noted the features of the multiactivity model. She claims this traditional model consists of short units of activity that permit minimal opportunities for sustained instruction and little transfer of learning across lessons, units, and year levels.

In a recent attempt to converge the learning and curriculum research agendas, Metzler (2005) proposed that physical educators adopt a models-based approach to teaching. Metzler (2005) presented a rationale for, and examples of, models-based instruction in physical education. The tactical games model (TGM) or teaching games for understanding (TGfU) is one such model (Griffin, Oslin, \& Mitchell, 1997). The TGM is an alternative to traditional, technique-led approaches to games teaching and learning and is an instructional model focused on developing learners' abilities to play games. The TGM approach assists players in learning the tactics and strategies of game play in tandem with technique development. All TGM teaching takes place within the framework of game play and modified game form, including modifications to rules, playing areas, and equipment. Techniques are developed using drills and other training practices common to the traditional, technique-led approach. A technique is introduced only when the players reach a level of game play that requires them to learn the technique. As the players' expertise develops, the game form is changed to continue to challenge the players in terms of game appreciation, tactical awareness, decision making, and execution of technique.

The TGM has been used extensively by teachers, and scholars have begun to develop a research base about how the model can be used effectively, as evidenced by a 2005 special edition of the journal Physical Education and Sport Pedagogy (Light, 2005) and an edited volume by Griffin and Butler (2005). In a review of some of the early studies of TGM, Rink, French, and Tjeerdsma (1996) note that TGM had produced a range of positive learning outcomes for students. The most powerful finding across these studies was that pupils who have experienced this model tend to perform better on tests of strategic knowledge than those who have been taught from a technique-based perspective. More recent studies of TGM, reviewed by Kirk and MacPhail (2000), have suggested that TGM may be perceived by pupils to be more enjoyable than the technique-based approach, and thus pupils may be more highly motivated to participate.

Kirk and MacPhail (2002) and Kirk and Macdonald (1998) argued that the model is compatible with cognitive and situated learning theories. From discussion of a situated learning perspective (Lave \& Wenger, 1991), learning is an active process of engagement with socially organized forms of subject matter, through perceptual and decision-making processes and the execution of appropriate movement responses. Individuals bring prior knowledge to learning episodes that contain a range of alternative conceptions of a topic. The learner's active engagement with subject matter is embedded within and is constituted by layers of physical, sociocultural, and institutional contexts. In a study of a modified basketball game, Kirk et al. (2000) proposed that there are at least three major interdependent dimensions of situativity. The first of these, a physical-perceptual dimension, concerns the physical environment (indoors/outdoors, surfaces, spaces, equipment, players) and the 
learner-players' interactions with this environment in terms of their perceptions. In the case of learning to play an invasion game, the physical-perceptual dimension refers to learner-players' abilities to perceive cues for interceptive actions (Davids, Savelsberg, Bennett, \& Van der Kamp, 2002), such as throwing a pass, cutting into space to receive a pass, catching a pass, closing down space, and intercepting a pass defensively. A second social-interactive dimension of situativity is concerned with the relational character of game play insofar as the performance of one player is highly dependent on the performance of other players. The social-interactive dimension also includes the informal social relations between learner-players because these social relations influence the nature of interaction between children (Carlson \& Hastie, 1997; Hastie \& Seidentop, 1999). A third dimension, the institutional-cultural, is concerned with the values of the school as an institution and aspects of popular physical culture that mediate young people's experiences of school physical education.

In another study of modified basketball, Rovegno, Nevett, Brock, and Barbiaz (2001b) applied a situated approach to identify physical-perceptual and socialinteractive dimensions of children's learning in physical education. Rovegno et al. (2001b) argued that within the traditional, multiactivity approach children tend to be taught to "pass and catch" in a static format. They suggest from a situated perspective children should be taught instead to make "catchable passes," involving interpretations and decisions concerned with physical-perceptual and social-interactive dimensions of games play, such as positions of players, speed of the pass, and the ability of the catcher.

In this article we were interested, in particular, in how young people learn to play games within a TGM approach (Griffin et al., 1997) in terms of the physicalperceptual and social-interactive dimensions of situativity. Building on the studies just mentioned, we utilized Kirk and MacPhail's (2002) development of the Bunker-Thorpe TGfU model to conceptualize the nature of situated learning in the context of learning to play an invasion game as part of a school physical education program. Kirk and MacPhail's adaptation highlights the importance of the relationships among thinking strategically, cue perception, and decision making, and to a lesser extent the relationships among decision making, technique selection, and movement execution. The modified game forms we developed and used in the study sought to represent and exaggerate (Holt, Strean, \& Bengoechea, 2002) specific strategies, cues, and decisions, and decisions, techniques, and movement.

The purpose of this study was to implement a "teaching experiment" (Rovegno et al., 2001a) in order to describe and analyze Year-5 (ages 9-10) children's learning in a physical education class. The substance of the teaching experiment was a modified version of a basketball/netball game based on the Invasion Game Unit 23 of the National Curriculum Physical Education (NCPE) in England and Wales. In this article, we will describe and analyze two specific, ubiquitous, and for some people elementary (or fundamental) skills of invasion game play-throwing and catching the ball. These skills can be viewed as elementary in terms of learning to play a modified game, and we are exploring the complex ways that students learn skills. We aim to examine the physical-perceptual and social-interactive dimensions of situated learning (Kirk et al., 2000) to explore in what ways these skills are complex, relational, and interdependent. 
We begin with an overview of the methods employed by this study, the context of the study, and the process of analyzing the data. In the main body of the article, we describe and analyze how children learned to throw and catch the ball in game situations involving attackers and defenders. The article concludes with some observations on the implications of the findings for constructing learning experiences using a TGM approach and assessment in physical education.

\section{Methodology}

A multiple-methods approach was used to collect and analyze data. Researchers have developed a number of approaches to measure learning progressions in the context of utilizing a TGM approach (Rink, et al. 1996; Oslin, Mitchell, \& Griffin, 1998). A recent approach developed by Brooker, Kirk, Braiuka, and Bransgrove (2000) and Rovegno et al. (2001b) involves the use of naturalistic observations and descriptions of game play, supplemented by naturalistic and semistructured individual and group interviews with learner-players and with teachers. The teaching experiment in the study presented here is not an intervention, but rather an investigation of situated learning in a modified game unit. Consequently, there is no attempt to compare two methods to demonstrate the superiority of one method over another.

\section{Context}

The study took place in a primary (elementary) school called Forest School (a pseudonym) in a town in the Midlands of England. The entire class of 29 Year5 students (ages 9-10 years) with an approximately equal number of boys and girls participated in a 12-lesson unit of an invasion game, involving two 40-min lessons per week for 6 weeks. The unit was based on Invasion Game Unit 23 of the National Curriculum for Physical Education (NCPE) for England and Wales designed specifically for this age group.

The classroom teacher-who had a specialist qualification in physical education as well as a primary school teaching qualification and several years of teaching experience-taught the unit. The teacher and one of the three members of the research team planned the unit jointly. Within the framework of the overall unit plan, individual lessons were planned by the teacher and researcher in light of a debriefing meeting immediately following each lesson.

\section{The Unit}

In this unit, children are to develop skilful attacking and team play. They are to learn how to work well as a team when attacking, and to explore a range of ways to defend. In all games activities, children are to think about how to use skills, strategies, and tactics to outwit the opposition. In invasion games, they enter their opponent's territory with the ball and try to get into good positions for shooting or reaching the goal. Details of this unit can be found by accessing the Department for Children, Schools, and Family (2007) Web site. 
In terms of previous learning, Invasion Game Unit 23 states it would be helpful for children to have developed passing and shooting skills, learned tactics for attacking, and followed rules in games. Lessons 1 through 4 in our unit involved preliminary tasks that provided us with baseline information on the learner-players' previous experiences and current competencies in relation to strategies, cues, and decisions, and decisions, techniques, and movements using 2 versus 1 and 3 versus 3 forms. Lessons 5 through 12 focused on the development of a limited number of offensive and defensive skills and techniques using 2 versus 1, 2 versus 2, 3 versus 1 , and 3 versus 3 forms. In this article, we focus primarily on the offensive skills of making catchable passes and cutting into space to receive a pass within the context of a modified game involving attackers and defenders. Specific information about the unit can be obtained by contacting the first author.

\section{Data Sources}

Each lesson was videotaped (except for Lesson 1, during which we had an equipment failure) using two cameras. Camera 1 followed the teacher, who wore a lapel microphone. The teacher's talk throughout each lesson was transcribed for analysis. Camera 2 focused on specific groups or games. For Lessons 5 through 12 , learner-players were grouped with other learner-players of the same or similar competencies demonstrated in Lessons 1 through 4. Two groups of three boys and two groups of three girls (i.e., target learner-players) who coped comfortably with the tasks in Lessons 1-4 were selected, and their game play was recorded for an approximately equal amount of time across Lessons 5-12. The written narratives of game play from Lesson 5 and Lesson 12 formed the primary data source for the principal analysis of learning progression recorded by Camera 2. The following video clips were selected for coding and analysis:

(a) Lesson 5:

Girls' Groups A and B, 2 versus 2

Boys' Groups A and B, 2 versus 2

(b) Lesson 12:

Girls' and boys' (mixed) Groups A and B, 3 versus 3

Two members of the research team and one experienced teacher who taught the class for 6 months immediately following the 12-lesson unit coded the clips from Lessons 5 and 12 independently. Their task was to write a detailed description of the game play in each case. The three coders then met and compared their descriptions. Reliability was determined by inclusion/omission of the key moments of game play in relation to the task set by the teacher (e.g., the cue "target hands," a cut into space, or an inappropriate defensive position). When a discrepancy between coders arose, the clips were viewed together and discussed. Even though the descriptions of some plays varied in terms of detail, in the case of all data coded in this way only minor amendments or additions were made in relation to key moments of game play. When the primary record of game play descriptions was formed, two members of the research team used the criteria and standards to make judgments 
about learning progressions between Lesson five and Lesson 12 . The data reported in this article draws on all three coders' accounts and contains all the key moments of game play noted by the coders.

Semistructured interviews were conducted with the teacher and selected students (six females, six males) from the target 3 versus 3 teams. The teacher was interviewed at the beginning of the unit, following each lesson, and at the end of the unit. Students were interviewed throughout the lessons specifically during game play. The purpose of the interviews was to gain insight into the participants' thinking and reflections on the unit. Typically, the questions were constructed around lesson events that had been observed and noted by the interviewer. A transcript of one postunit semistructured interview with the teacher supplements the written narrative descriptions of game play and the Game Performance Assessment Instrument (GPAI) data.

The GPAI was used to measure game play performance of the six target learner-players. The GPAI is a valid and reliable instrument that can be used to measure individual components of game performance (Griffin et al., 1997; Oslin, et al., 1998). The GPAI was designed to provide teachers and researchers with a means of observing and coding performance behaviors that demonstrate the ability to solve tactical problems in games by making decisions, moving appropriately, and executing skills. The GPAI contains seven components (base, adjust, decision made, skill execution, support, cover, mark/guard) of game performance-some or all of which may apply to a particular game, lesson, or unit. The components of the GPAI used to measure game performance consisted of (a) decision made, (b) skill execution, (c) support, and (d) mark/guard (see Table 1). The components measured represent the content of the unit (i.e., tactical problems that pupils solved). The six target pupils were videotaped during Lessons 5 and 12, and the following indices were used to measure performance.

\section{Table 1 GPAI Criteria}

\begin{tabular}{|c|c|}
\hline GPAl component & Criteria \\
\hline \multirow[t]{2}{*}{ Decision made } & Player attempts to pass to an open teammate. \\
\hline & Player attempts to shoot when open. \\
\hline \multirow[t]{5}{*}{ Skill execution } & Reception \\
\hline & - $\quad$ Target hands \\
\hline & - Control ball \\
\hline & Passing \\
\hline & - $\quad$ Ball to target \\
\hline Support & $\begin{array}{l}\text { Player appeared to support ball carrier by being in or moving to } \\
\text { an appropriate position to receive pass. }\end{array}$ \\
\hline Mark & $\begin{array}{l}\text { Player appeared to take the opportunity to mark/guard a player } \\
\text { with or without the ball (opportunity to mark). }\end{array}$ \\
\hline
\end{tabular}


- Decision Made Index (DMI): (number of appropriate decisions made) $\div$ [(number of appropriate decisions made) + (number of inappropriate decisions made)]

- Skill Execution Index (SEI): (number of efficient skill executions) $\div$ [(number of efficient skill executions) + (number of inefficient skill executions)]

- Support Index (SI): (number of appropriate supporting movements) $\div$ [(number of appropriate supporting movements) + (number of inappropriate supporting movements)]

- Mark/Guard Index (MI): (number of appropriate marking movements) $\div$ [(number of appropriate marking movements) + (number of inappropriate marking movements)]

- Game Performance Index (GPI): (DMI + SEI + SI + MI $\div 4$

When scoring the individual components, a score greater than 1 indicates that a player performed more appropriate/efficient than inappropriate/inefficient. The use of the indices prevents a score of negative value, which might prevent teachers from using this measure.

In the study, game play data were collected from videotapes of the six target pupils in Lessons 5 and 12 using the criteria shown in Table 1. During the game play episodes, the participants played in a 3 versus 3 half-court netball game of 7 min in duration (used running time) to ensure that all participants had equal playing time. Two coders were used, both of whom were trained in using the GPAI. Training for the coders continued until they consistently exceeded interobserver agreement of .80 . The overall average measure for the interobserver reliability for the GPAI was .94.

We acknowledge that GPAI data is more likely to be gathered in primary schools by researchers pursuing studies similar to the one reported in this article than by classroom (generalist) teachers of physical education. However, this does not detract from its refined specific use by students and teachers. For example, students can use one or two components of the GPAI to assess peers or themselves. Teachers (either watching live play or recordings of game play) can use it as they observe students in game play as a means of formative and summative assessment.

\section{Findings and Discussion}

\section{Throwing and Catching as Relational Skills}

We focus on two aspects of game play that featured prominently throughout the unit: passing and movement into space to receive a pass within the context of a modified game involving attacking and defensive play. We illustrate, following Rovegno et al. (2001b, p. 386), that these two aspects of game play are relational, in the sense that "immature performance does not reside solely in the individual but in the relation between passer and receiver" and, we add, in the relationships among passers/receivers and opponents. We show that learning to throw and catch involves players' interpreting, constructing, and responding to the physical-perceptual and social-interactive dimensions of the learning environment. 


\section{Throwing a Catchable Pass}

Traditional practice in physical education lessons has tended to view skill development as synonymous with and limited to improving the technical expertise of individual learner-players. Lessons informed by this perspective often feature decontextualized and individualized technique practices and drills (Kirk \& MacPhail, 2002; Thorpe \& Bunker, 1989). As Thorpe and Bunker (1989) stressed, this is an impoverished view of skill development. They emphasize instead that individual technique is only one part of a skill. The other key dimension of skillful performance is the effective and appropriate execution of techniques within a game. Partially reflecting the traditional perspective, the teacher commented that the technical aspects of passing were dependent on individual developmental characteristics, such as previous experience, physical size, and amount of practice time:

Skill development I think we still approached in two ways, in that you naturally did have to practice the skills and we did set time apart to practice specific skills because I don't think they'd had that much time in throwing and passing so I think some of them needed the time to develop. I don't think their skills were that strong so some of them really needed that time to develop the skills. (teacher, postunit interview)

But she also recognized the interpretative and relational character of making catchable passes, as she went on to say:

Some of them (learner-players) obviously were naturally very competent but there were a few that struggled to get an accurate pass to the person they wanted it to go to and there was a big difference in the group in being able to execute those skills quickly before the person they were trying to pass to was being marked again. (teacher, postunit interview)

What the teacher signals in the latter comment is her understanding that the skill of passing includes perceptual and decision-making dimensions. This means that a catchable pass was not solely a matter of individual competence; much depended on the off-the-ball movement of fellow players and the passing player's ability to read and anticipate their movements within the context of the game form and the physical spaces it constructs.

The TGM approach recognizes both aspects of skill development, although it stresses the second characteristic through its emphasis on the extensive use of modified games. In the 2 versus 2 game form, attackers attempted to score by hitting with the ball a target on a wall within a relatively small defined space. When possession was turned over, play restarted from an end line opposite the target and the roles of attackers and defenders switched. Defenders were instructed to mark one player and stay with that player throughout a play, thereby reducing the potential complexity of the social-interactive dimension of the situation. Within this physical environment, the attacking player off the ball had to get free from her or his marker in order to receive a pass. The perceptual challenge for the attackers was for the off-the-ball player to use space appropriately and for the player on the ball to recognize the cue "target hands," to be shown by the player off the ball 
when s/he was in space. However, the task of getting into space and sending an appropriately timed, weighted, and directed pass under the pressure provided by defenders proved to be challenging for all learner-players.

Early in the unit, such as in Lesson 5, learner-players tended to use long, looping passes most frequently, a point noted by the teacher:

They still tended to use the loopy one too much, because they thought that there was somebody there in front of them and they'd got to get it over them. ... [They] thought that's the person I want to pass it to, there's somebody in the way so I'm just going to go over the top and hope that they can catch it. And because there was somebody in the way they didn't necessarily have the skill to be accurate in the loopy passes. (teacher, post unit interview)

This overuse of the looping pass, as the teacher notes, was most often due to the positioning of the receiver in the 2 versus 2 game forms, who regularly stood behind defenders. For example, during one play in Lesson 5, the receiver makes a "banana cut" behind the defenders. Throughout her cut she has her hands up in the target hands cue position and she remains behind the two defenders, who merely have to slide to the side to maintain their defensive positions. The receiver makes her banana cut across the court and back again to rest where she had started, while her teammate with the ball has remained in the ready-to-throw position throughout.

As Rovegno et al. (2001b) comment, the banana cut reduces the perceptual demands of receiving the ball because the receiver maintains the same distance from the passer throughout the movement. But this kind of off-the-ball movement is relatively easy to defend. With no other option available to her, the player in possession threw a looping pass over the heads of the defenders, which in this case resulted in a rare catch and score, a point we will return to momentarily.

\section{Better Movement Makes Passing Easier}

As the unit progressed, learner-players were gradually able to make more effective passes because of better movement into space and sharper cuts. They were able to identify, when asked, that the quality of passing and movement of players signified good play:

Interviewer: Good, that was excellent play. Why was it good?

Pupil: Because nobody marked Sarah and she was. (inaudible)

Interviewer: OK, so you're missing a marker, but what else made it good? What about passes?

Pupil: Short.

Interviewer: They were short and what?

Pupil: Quick.

Interviewer: Excellent. Why short passes?

Pupil: Because they're much easier to get (to catch). (Pupil interviews, Lesson 12) 
At the same time, although some learner-players could recognize the importance of short, quick passes and the use of the looping pass became less frequent, the learner-players remained reluctant to use the variety of passes they had been taught. The teacher commented:

It was really nice seeing that there was an improvement, but they still didn't use the bounce passes or a variety of passes that I'd have liked to see them doing but that's a variety of skill that I think will develop with their confidence. The ability to be able to anticipate which way their teammate was going to go and to be able to pass ahead of them, obviously the better groups were beginning to do that. (teacher, postunit interview)

Given the perceptual and relational demands of the 2 versus 2 and 3 versus 3 game formats, where much of the players' attention was directed to cue recognition and movement off the ball, the expectation that they might use a variety of passes was perhaps unrealistic at this stage.

The looping pass did persist, however, when there was inappropriate off-the-ball play. For example, during a play in a 3 versus 3 format in Lesson 12, the looping pass over the heads of defenders was used twice in succession, once effectively in terms of retaining possession and once ineffectively in terms of turning over possession. In the case of the second outcome, it is slow decision making on the part of the player in possession that allows his teammate to be marked, which then elicited the careless, one-handed loop pass and the turnover.

The GPAI results support the target learner-player improvement with regard to passing as an interpretative and relational skill. With regard to the specific on-theball skills (i.e., passing criteria $=$ ball to target and receiving criteria = target hands; control ball) and off-the-ball movements (i.e., support criteria = player appeared to support ball carrier by being in or moving to an appropriate position to receive pass), learner-players showed improvement in solving the tactical problem of maintaining possession of the ball. Skill execution improved from .63 to .76 and support improved from .58 to .61 . The target learner-players also showed improvement in decisions made (i.e., player attempts to pass to an open teammate) from .58 to .71 . The target learner-players' overall offensive game performance measures, (DMI + $\mathrm{SEI}+\mathrm{SI}) \div 3$, showed improvement from .59 to .69 .

\section{Cue Perception as a Relational Skill}

Holding on to the ball too long is according to Rovegno et al. (2001b) a common feature of immature game play. Taking a long time to decide what to do when onthe-ball was a feature of game play at Forest School among the less experienced and able learner-players. The teacher felt that lack of experience in game play, an associated lack of confidence, and inability or reluctance to become fully involved in the game were key factors behind slow decision making on-the-ball. She commented:

I think those children hadn't played those kind of games before and if they had they'd been in a big game situation and probably only received one pass in the whole game. I mean I'm guessing because I hadn't seen them before that but I think that's where the lack of confidence comes from. (teacher, postunit interview) 
Holding the ball too long also occurred among the able learner-players. However, it is clear from the following examples that individual experience and confidence are not the only factors affecting the length of time the player in possession holds the ball. For instance, in a play from Lesson 5 we described earlier, it is clear that the length of time the player in possession holds the ball is very much a function of the inappropriate use of the target hands cue and positioning of her teammate. This same problem was also evident in plays from other lessons.

In a play from Lesson 12 (3 versus 3 game), for example, there is very little movement from the attackers off-the-ball, making defense easier. This evidence suggests that in a modified game situation holding the ball too long is in part influenced by individual player experience and confidence, but also by the actions of other players on the court, supporting the notion that allegedly simple skills such as passing are relational and complex.

As the unit progressed, learner-players became better at using and recognizing appropriate cues for making and receiving a pass, thereby improving the speed of decision making. In the 2 versus 2 format in Lesson 5, we have reported that long looping passes predominated in large part because receivers positioned themselves behind defenders and made side-slipping banana cuts. Sometimes, receivers used the target hands cue at inappropriate times, such as when they were not free to receive a pass.

The play from Lesson 5 illustrates the consequences of the misuse of cues for the passer. In this case, the player in possession used a cue-the defenders looking away from the ball and behind them for the receiver-to time her pass. While in this case the pass was caught and a point scored, this was not consistently performed because the attacking team could not count on the defender to behave in this way regularly. Indeed, on the next attacking play by the same attacking team, the defenders did not oblige by looking away with a predictable result because the pass of the player in possession was easily intercepted by a defender.

The 3 versus 3 full-court game was considerably more complex than the 2 versus 2 half-court game because a turnover in possession resulted in defensive and attacking roles changing immediately. Regular turnover meant there was less time for the team who turned over possession to reset their defense. The larger playing area and the targets at both ends of the court created new perceptual and relational challenges for players, and increased both the distance over which the ball could travel and higher speeds in terms of movement of players. Interestingly, and echoing a point noted by Rovegno et al. (2001b), the more complex environment elicited more mature responses from the learner-players, even though these were not always completed successfully.

For example, evidence of improved cue use and recognition can be found in some clips from Lesson 12 during a 3 versus 3 full-court game. In these cases, there was some evidence of learning progression in terms of appropriate use and quick recognition of the target hands cue in conjunction with sharp cuts into space. When cues were recognized quickly, typically the ball was moved quickly and more often than not the defense beaten. Here, effective and appropriate cue use and perception is relational in terms of the attackers' abilities to work off each other's cues, which in turn depends on the defenders' actions.

Indeed, we recorded that it was an attempt by a defender, who had been marking his opponent very closely, to pick up another attacker who had made a sharp cut 
that permitted a crucial breakthrough to occur and lead to a score. Even though the defense had appeared to be effective, with the defender demonstrating a good body position, facing his opponent, and between his opponent and the passer, a good cut by one attacker off-the-ball allowed the second off-the-ball attacker to move into space and opened up the defense. The speed of this attacking move, particularly in terms of the speed of movement of the ball, was impressive. But because this kind of play was complex, requiring appropriate and effective use and perception of cues by attackers and defenders, instances of game play at this level remained infrequent. More often, owing to the complexity of cue use and perception, play more often resulted in intercepted passes and regular turnovers.

According to an analysis of game play from a traditional perspective, attacks tend to break down and possession is turned over because of poor individual passing technique. An alternative reading of game play from a relational and situated perspective suggests that at least as important are learner-players' abilities to recognize appropriate cues, and the impact on cue use and perception of the actions of both teammates and opponents.

GPAI data from Lessons 5 and 12 showed improvements in offensive play; however, results indicated a decrease in defensive play as it relates to marking/guarding from .74 to .38 . The decrease in marking/guarding and overall game performance (.63 to .58) reflects the change in the game from half-court to full-court games. Learner-players struggled to make smooth transitions from offense to defense, often losing track of the player they were to mark/guard and simply playing the ball. By playing the ball, defenders left offensive players open to make successful movement down the court to set up for a shot.

\section{Implications for Making Judgments about Learning Progression}

At the end of the unit, the teacher reflected that "they've all improved, which I was pleased about; I'd say that's the most important thing" (teacher, postunit interview). We shared the teacher's view that there was some evidence of learning progression in relation to specific aspects of game play, such as passing, receiving, and defending between the first and second half of the unit. For example, toward the end of the unit, learner-players showed target hands with increasing appropriateness. Key features of game play in the second half of the unit compared with the first four or five lessons, specifically for the groups of learner-players we recorded, were increased speed of the game, increased speed the ball traveled, and increased pace of movement off-the-ball. But the quality of game play was highly volatile and inconsistent. Supporting Metzler (2005), the teacher suggested that 12 lessons was too short a time for many of the children who entered the unit with little experience of playing an invasion game like this to make significant progress:

We were telling them to look for the first movement and as soon as they saw them moving they should be ready to pass. I don't think they necessarily got there but I think there was an improvement. Some of them at the beginning were really lost for where to pass or who to pass to or being aware of that and they did start trying to look I think just perhaps it wasn't long enough. (teacher, postunit interview) 
She also supported a commonplace finding in the TGM literature (Rovengo et al., 2001a) that declarative knowledge of what to do often seems to develop in advance of actual game performance: "I just feel so frustrated for them because you can see what they want to do but it doesn't work and you can see that they're trying very hard to get into a position to get a pass" (teacher, postunit interview).

The teacher and the researchers were able to make general judgments about learning progression. But it was quite another matter to try to make a definitive assessment of learning in relation to individual players. The evidence from this study shows it is extremely difficult to make judgments about specific learner-players, which is the conventional practice in school physical education. It is possible to make judgments about individuals' techniques for passing, for example, when the technique is isolated from the game context, which is why we think individual drill-like practices have persisted in physical education for so long. As the TGM literature shows (Rovengo et al., 2001b), however, throwing a pass in a decontextualized practice with good technique is different from throwing a catchable pass. Because passing and receiving are shaped by the physical-perceptual and socialinteractive dimensions of the learning situation, they are, as we along with Rovegno et al. (2001b) have argued, relational skills. As such, each individual's performance and opportunities to learn are crucially affected by the capacities and motivation of other players and their interpretations of and contributions to the game.

Grouping players according to experience and ability and matching task difficulty to ability groups facilitate the management of these difficulties of assessment. But the terms experience and ability are themselves highly problematic because how we determine what they mean in a specific context with a specific group of learner-players is precisely the problem we are wrestling with, in terms of making judgments about individuals' learning progressions (Evans, 2004).

We propose that the evidence presented in this article on the relational character of such relatively elementary skills as passing and receiving supports a TGM approach to games teaching because the use of modified games as the primary pedagogical strategy recognizes relationality as a key feature of game play. The TGM approach also recognizes implicitly, although the issue has rarely been addressed in the TGM literature, that the modified game itself rather than individual learnerplayers or teams should properly be the unit of analysis in making judgments about learning progression. Because the game sets the problem in a TGM unit, it is important to consider the appropriateness of the modified game as learning progresses. Game play is a dynamic event that requires skill proficiency as well as tactical understanding. We feel, however, that this proposal presents far-reaching challenges for conventional practices of assessing learning in games that remain steadfastly focused on the individual (Piotrowski \& Capel, 2000).

\section{Conclusion}

The focus for this study was two elementary, or fundamental, skills of invasion game play - throwing and catching a ball. We suggested that these skills are sometimes viewed as simple and elementary aspects of physical education programs. We sought to explore the complex ways that students learn skills. We examined the 
physical-perceptual and social-interactive dimensions of situated learning (Kirk et al., 2000) to show that for players who are in the early stages of learning to play a ball game, these skills are complex, relational, and interdependent.

This exploration of throwing and catching was located in two contexts. The first of these was within a theoretical context, with a view to refining and extending aspects of situated learning theory in particular, although we also acknowledged the influence of cognitive learning theory on our thinking about learning in physical education. In this respect, we used two dimensions of situativity identified by Kirk et al. (2000) and Rovegno et al. (2001a), the physical-perceptual and socialinteractive dimensions, to inform our work in constructing learning experiences for the learner-players, and to aid our analysis of the data.

We think this approach has produced promising results in at least two ways. First, we were able to use these concepts to manipulate the physical spaces and the relationships of the players, and to represent and exaggerate the strategies, cues, and decisions, and decisions, techniques, and movement aspects of game play. Second, we were able to explain some of the difficulties and challenges beginning players encounter when learning to play a ball game. In particular, this study sheds further light on Rovegno et al.'s (2001b) claim that so-called elementary skills such as throwing and catching are complex and difficult to learn because they are relational and interdependent. We propose that the three dimensions of situativity may be constructs that are robust enough to warrant further testing and refinement in physical education and youth sport settings.

The second context in which we located this exploration of throwing and catching was a models-based approach to games teaching, focused around the well-researched and established TGM. It was noted in the introduction that the compatibility of cognitive and situated learning theories and the TGM had already been proposed (Kirk \& Macdonald, 1998; Kirk \& MacPhail, 2002). We suggest that this compatibility is further evidenced by this study.

There is a further aspect of approaching throwing and catching from a TGM perspective that is important in terms of understanding theory and practice. Modelsbased instruction starts from the identification of learning outcomes within broader educational aspirations and values, and then works back to determine the best instructional strategies and subject matter to achieve these outcomes. This focus on outcomes inevitably and properly leads to posing questions about the extent to which the outcomes have been achieved. We used several sources of data to interpret and make judgments about the players' learning progressions. We acknowledge that the likelihood of classroom (generalist) or even specialist teachers of physical education committing to both a TGM pedagogy and to GPAI assessment techniques is low. In our use of several sources of data, and in generating data that supported the notion of throwing and catching as relational skills, we began to realize that it is difficult and possibly inappropriate to take the individual as the unit of analysis when assessing learning progression. While exploring the implications of the study for assessment in physical education, we were led to the intriguing idea that perhaps the most appropriate unit of analysis in games programs should be the game itself. We think this issue is also worth exploring further because it may have radical implications for how physical educators make judgments and record the learning progressions of their students. 


\section{References}

Brooker, R., Kirk, D., Braiuka, S., \& Bransgrove, A. (2000). Implementing a Game Sense approach to teaching year 8 basketball. European Physical Education Review, 6, 726.

Carlson, T.B., \& Hastie, P.A. (1997). The student social system within sport education. Journal of Teaching in Physical Education, 16, 176-195.

Cobb, P., \& Bowers, J. (1999). Cognitive and situated learning perspectives in theory and practice. Educational Researcher, 28, 4-15.

Davids, K., Savelsberg, G., Bennett, S.J., \& van der Kamp, J. (2002). Interceptive actions in sport: theoretical perspectives and practical applications. In K. Davids, G. Savelsberg, S.J. Bennett, \& J. van der Kamp (Eds.), Interceptive actions in sport: Information and movement (pp. 1-39). London: Routledge.

Department for Children, Schools and Family (2007). The Standards Site. Retrieved October 5, 2007, from http://www.standards.dfes.gov.uk/schemes2/phe/?view = get

Dodds, P., Griffin, L.L., \& Placek, J.L. (2001). A selected review of the literature on development of learners' domain-specific knowledge. Journal of Teaching in Physical Education, 20, 301-313.

Ennis, C.D. (1999). Creating a culturally relevant curriculum for disengaged girls. Sport, Education and Society, 4, 31-50.

Evans, J. (2004). Making a difference? Education and ability in physical education. European Physical Education Review, 10, 95-108.

French, K.E., \& McPherson, S.L. (1999). Adaptations in response selection processes used during sport competition with increasing age and expertise. International Journal of Sport Psychology, 30, 173-193.

Griffin, L.L, \& Butler, J.I. (2005). Teaching Games for Understanding: Theory, research, and practice. Champaign, IL: Human Kinetics.

Griffin, L.L., Oslin, J.L., \& Mitchell, S.A. (1997). Teaching sports concepts and skills: A tactical games approach. Champaign, IL: Human Kinetics.

Hastie, P.A., \& Siedentop, D. (1999). An ecological perspective on physical education. European Physical Education Review, 5, 9-29.

Holt, N.L., Strean, W.B., \& Bengoechea, E.G. (2002). Expanding the teaching games for understanding model: new avenues for future research and practice. Journal of Teaching in Physical Education, 21, 162-176.

Kirk, D., Brooker, R., \& Braiuka, S. (2000, April). Teaching Games for Understanding: A situated perspective on student learning. Paper presented at the annual meeting of the American Educational Research Association, New Orleans, LA.

Kirk, D., \& Macdonald, D. (1998). Situated learning in physical education. Journal of Teaching in Physical Education, 17, 376-387.

Kirk, D., \& MacPhail, A. (2002). Teaching games for understanding and situated learning: Re-thinking the Bunker-Thorpe Model. Journal of Teaching in Physical Education, 21, 177-192.

Kirk, D., \& MacPhail, A. (2000). The Games Sense Approach: Rationale, description, and a brief overview of research. Leeds, U.K.: Human Kinetics.

Lave, J., \& Wenger, E. (1991). Situated learning: Legitimate peripheral participation. New York: Cambridge University Press.

Light, R. (2005). Special issue: Teaching Games for Understanding. Physical Education and Sport Pedagogy, 11, 211-212.

Metzler, M.W. (2005). Instructional models for physical education (2nd ed.). Boston: Allyn and Bacon. 
Oslin, J.L., Mitchell, S.A., \& Griffin, L.L. (1998). The Game Performance Assessment Instrument (GPAI): Development and preliminary validation. Journal of Teaching in Physical Education, 17, 231-243.

Piotrowski, S., \& Capel, S. (2000). Formal and informal modes of assessment in Physical Education. In S. Piotrowski \& S. Capel (Eds.), Issues in Physical Education (pp. 99114). London: Routledge/Falmer.

Rink, J.E. (1999). Instruction from a learning perspective. In C.A. Hardy \& M. Mawer (Eds.), Learning and Teaching in Physical Education (pp. 149-168). London: Falmer.

Rink, J.E., French, K.E., \& Tjeerdsma, B.L. (1996). Foundations for the learning and instruction of sport and games. Journal of Teaching in Physical Education, 15, 399-417.

Rovegno, I., Nevett, M., \& Babiarz, M. (2001a). A field-based project on learning and teaching of invasion game tactics in fourth grade: Introduction and theoretical perspective. Journal of Teaching in Physical Education, 20, 341-351.

Rovegno, I., Nevett, M., Brock, S., \& Barbiaz, M. (2001b). Teaching and learning of basic invasion game tactics in fourth grade: A descriptive study from a situated theoretical perspective. Journal of Teaching in Physical Education, 20, 370-388.

Thorpe, R., \& Bunker, D. (1989). A changing focus in games education. In L. Almond (Ed.), The place of physical education in schools (pp. 42-71). London: Kogan Page. 
Copyright of Journal of Teaching in Physical Education is the property of Human Kinetics Publishers. Inc. and its content may not be copied or emailed to multiple sites or posted to a listserv without the copyright holder's express written permission. However, users may print, download, or email articles for individual use. 